\title{
Sphaleron rate in lattice gluodynamics
}

\author{
A.Yu. Kotov* \\ Moscow Institute of Physics and Technology, Dolgoprudny, 141700 Russia \\ Institute for Theoretical and Experimental Physics NRC "Kurchatov Institute", Moscow, 117218 \\ Russia \\ Bogoliubov Laboratory of Theoretical Physics, Joint Institute for Nuclear Research, Dubna, \\ 141980 Russia \\ E-mail: kotoveitep.ru
}

QCD vacuum can be treated as a series of energetically equivalent, but topologically distinct sectors. The rate of transitions between various topological sectors is determined by the sphaleron rate $\Gamma_{C S}$. This quantity is given by the limits of zero frequency and zero momentum of the correlator of topological charge density. Sphaleron rate is very similar to such transport coefficients as viscosity or conductivity. Sphaleron rate also describes time relaxation of the chiral charge. In this report we discuss the measurement of sphaleron rate in lattice gluodynamics in the deconfinement phase. We use Gradient Flow to measure the topological charge density correlator, and we also take continuum limit. We provide an estimation of the sphaleron rate in lattice gluodynamics and compare it with results obtained by other methods.

XIII Quark Confinement and the Hadron Spectrum - Confinement2018

31 July - 6 August 2018

Maynooth University, Ireland

* Speaker. 


\section{Introduction}

It is well known that Yang-Mills theory possesses infinite series of energetically degenerate but topologically distinct vacua[1,2], characterized by an integer, Chern-Simons number $N_{C S}$. Transitions between various sectors can occur due to the tunneling process, instanton[3]. At finite temperature thermal fluctuations of the gluonic field (exciting unstable field configurations sphalerons) also can change the Chern-Simons number and lead to the transition to another sector. The evolution of the Chern-Simons number with time $t$ is a random walk with a rate $\Gamma_{C S}$ given by the sphaleron transitions:

$$
\Gamma_{C S}(t)=\lim _{t \rightarrow 0} \frac{\left\langle\Delta N_{C S}^{2}(t)\right\rangle}{V t}
$$

In a theory with fermions due to anomaly the change of Chern-Simons number is accompanied by the change of a corresponding fermionic charge. For example, in electroweak theory the sphaleron transitions explain baryon number nonconservation. In Quantum Chromodynamics, the sphaleron transitions lead to the change of quark chirality.

Let us overview, what is known about the value of the sphaleron rate in QCD and QCD-like theories. In the approximation of high temperature $T$ and in the weak coupling regime $\Gamma_{C S} \sim$ $\alpha^{5} \log 1 / \alpha T^{4}[4]$, where $\alpha$ is the coupling constant. Numerical simulations in the weak-coupling regime and attempts to extrapolate the result to the temperatures close to the confinement - deconfinement phase transition give[5]: $\Gamma_{C S} \sim 30 \alpha^{4} T^{4}$. It is also worth mentioning the results for $\mathscr{N}=4$ Super Yang-Mills, obtained by holographic methods: $\Gamma=\frac{g^{2} N}{256 \pi^{3}} T^{4}[6]$. Improved holographic QCD, which corresponds to the gluodynamics in the limit of large number of colors, gives much larger result[7]. For example, at the critical temperature $\Gamma\left(T_{c}\right) / T_{c}^{4}>1.64$. In [8], the first attempt to measure the sphaleron rate in lattice $S U(2)$ gluodynamics was performed, where the authors obtained $\Gamma / T^{4} \sim 10^{-3}$. It can be easily seen that the results within various approaches are rather controversial. Nonperturbative evalution of the sphaleron rate in QCD nowadays is absent. For this reason in this report we would like to study the sphaleron rate by means of lattice simulations, which is one of the most powerful approaches for studying nonperturbative phenomena. Despite a considerable progress in the lattice study of QCD and other theories, today it is not possible to calculate the sphaleron rate in the theory with fermions. For this reason, in this report we consider the sphaleron rate in gluodynamics, in the vicinity of the confinement-deconfinement phase transition.

\section{Lattice setup}

Since the change of the Chern-Simons number is given by the integral of the topological charge $q(x)=\frac{g^{2}}{32 \pi^{2}} F_{\mu v}^{a} \tilde{F}_{a}^{\mu v}: \Delta N_{C S}(t)=\int d^{3} x \int_{0}^{t} q(x, t)$, the sphaleron rate $\Gamma_{C S}$ is the zero frequency limit of the retarded Green function $\operatorname{im} G^{R}(\omega, \vec{k}=0)$ of the topological charge density:

$$
\begin{aligned}
\Gamma_{C S} & =-2 \pi T \lim _{\omega \rightarrow 0} \frac{\rho(\omega)}{\omega}, \\
\rho(\omega) & =\frac{1}{\pi} \operatorname{im} G^{R}(\omega, \vec{k}=0)
\end{aligned}
$$

Here $\rho(\omega)$ is the spectral function of the correlator of the topological charge density. The minus sign in front of (2.1) appears due to the pseudoscalar nature of the topological charge density 
operator. Since simulations on the lattice are performed in Euclidean space, one has to make an analytical continuation. In Euclidean space the correlator is related to the spectral function via the integral relation:

$$
G_{E}(t)=\int_{0}^{\infty} \rho(\omega) \frac{\cosh \omega(\beta / 2-t)}{\sinh \omega \beta / 2} d \omega
$$

Lattice study of the sphaleron rate is analagous to the investigation of such transport coefficients as electric conductivity $[9,10]$, shear viscosity $[11,12,13]$, bulk viscosity $[14,15]$ and others.

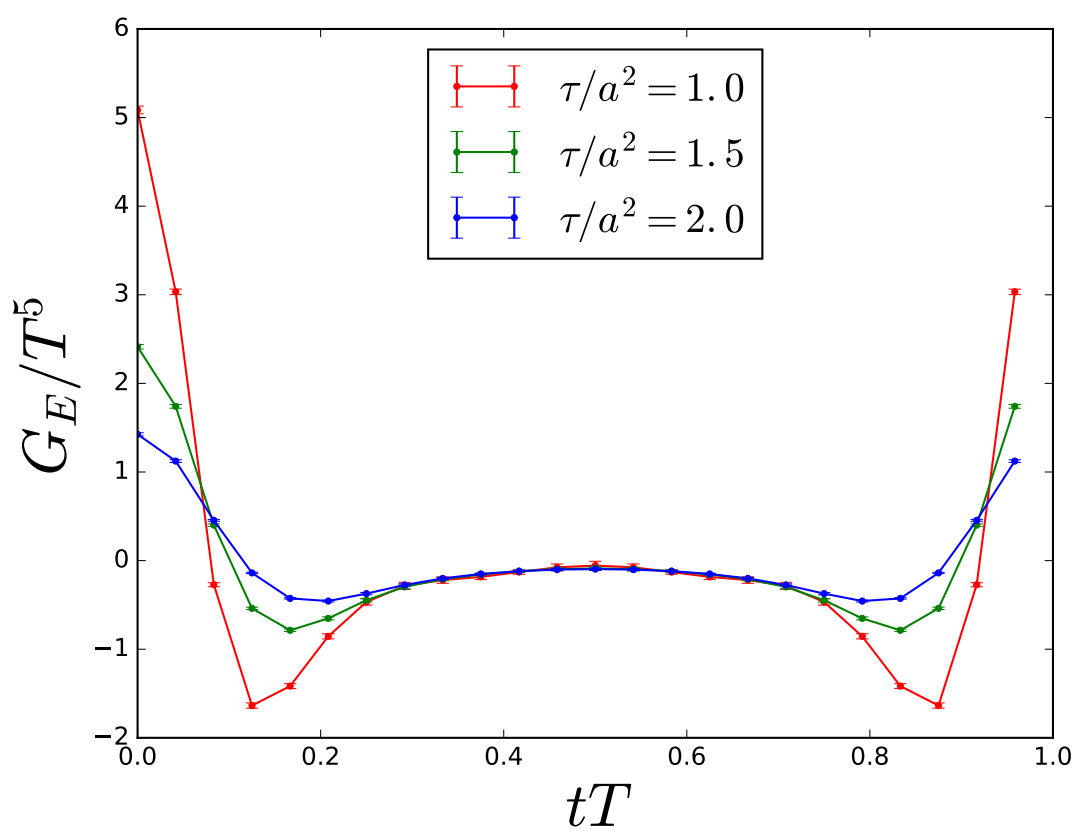

Figure 1: Topological charge density correlator for the temperature $T / T_{c}=1.24$, the lattice size $24 \times 70^{3}$ and three values of the GF time $\tau / a^{2}=1.0,1.5,2.0$.

Numerical simulations were performed in gluodynamics with gauge group $S U(3)$ and Wilson gauge action. To measure the topological charge density and its correlator, the Gradient Flow method[16] was applied. Using Gradient Flow one can measure renormalized correlator $C_{q}(t)$ of the topological charge density[17] using:

$$
C_{q}(x)=\lim _{\tau \rightarrow 0} \lim _{a \rightarrow 0}\left\langle q_{L}^{\tau}(x) q_{L}^{\tau}(0)\right\rangle,
$$

where $q_{L}^{\tau}(x)$ is the discretized operator of the topological charge density measured at the Gradient Flow time $\tau$. Moreover the behaviour of the corrections at nonzero $\tau$ and $a$ is also known[17]:

$$
\left\langle q_{L}^{\tau}(x) q_{L}^{\tau}(0)\right\rangle=C_{q}(x)+O_{\tau}\left(a^{2}\right)+O(\tau)
$$

Note that discretization errors $O_{\tau}\left(a^{2}\right)$ in general depend on $\tau$, so the order of limits in Eq. (2.3) is important. One should take continuum limit first and then set $\tau \rightarrow 0$. 


\section{Results}

We present the results for three temperatures $T / T_{c}=1.24,1.50,1.70$. For each temperature to take the continuum extrapolation we performed measurements at four lattice sizes $12 \times 36^{3}$, $16 \times 48^{3}, 20 \times 60^{3}, 24 \times 70^{3}$. To check finite volume effects, for $T / T_{c}=1.24$ simulations at lattice sizes $16 \times 32^{3}$ and $16 \times 64^{3}$ were also performed. The dependence of the lattice step on bare parameter $\beta$ was taken from[18].

An example of the topological charge density correlator is presented in Fig. 1. On this figure the correlator for the temperature $T / T_{c}=1.24$, the lattice size $24 \times 70^{3}$ and three values of the GF time $\tau / a^{2}=1.0,1.5,2.0$ is shown. One sees that for the middle points the correlator is negative as expected. For small values of Euclidean time $t$ the correlator becomes positive due to the large positive contact term which gets smeared at nonzero values of $\tau$. For that reason one should omit points with values of $t$ smaller than the characteristic radius of smearing $\sim \sqrt{8 \tau}$. At $\tau \lesssim a^{2}$ the discretization errors might be large. For this reason in the following we use data with $t^{2} / 8 \gtrsim \tau \gtrsim a^{2}$ for the extrapolation.

We have found that the $a$-dependence of the correlator for all values of GF time $\tau$ and Euclidean time $t$ within the restrictions discussed above can be described by $a^{2}$ term. For example in the Fig. 2 we present the correlators for $\tau / a_{24}^{2}=4, t / a_{24}=12$ and $\tau / a_{24}^{2}=1.5, t / a_{24}=7$ (the data is for the temperature $T / T_{c}=1.24, a_{24}$ is the lattice step for the lattice size $24 \times 70^{3}$ ) as a function of $1 / N_{t}^{2} \sim a^{2}$. For $\tau / a_{24}^{2}=1.5$ only three points are presented, because the lattice step $a_{12}$ for the lattice $12 \times 36^{3}$ does not satisfy the restriction $\tau \gtrsim a^{2}$. Also we have seen that the results for $16 \times 32^{3}, 16 \times 48^{3}$ and $16 \times 64^{3}$ coincide within errorbars, thus finite volume effects are negligible.
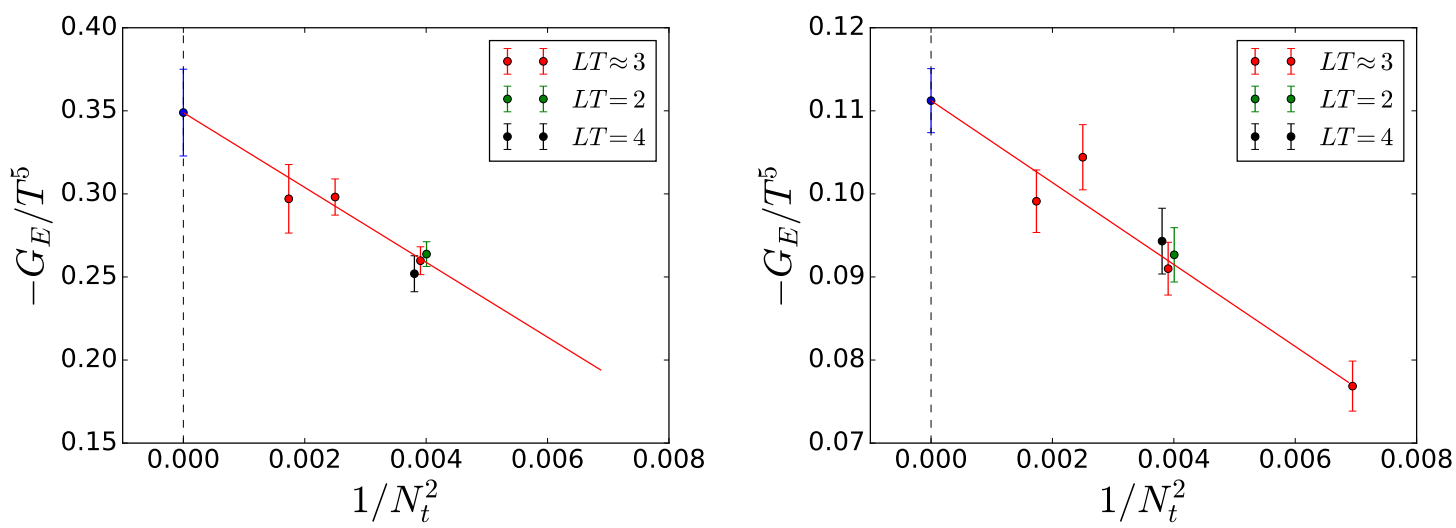

Figure 2: The dependence of the correlator $-G_{E} / T^{5}$ on the $1 / N_{t}^{2}$ for $\tau / a_{24}^{2}=1.5, t / a_{24}=7$ (left) and $\tau / a_{24}^{2}=4, t / a_{24}=12$ (right). Temperature is $T / T_{c}=1.24$. Lines correspond to the quadratic fit $G_{E}=$ $G_{E}(a=0)+C a^{2}$. Points for lattice sizes $16 \times 32^{3}$ and $16 \times 64^{3}$ are also shown.

Using the results of the extrapolation $a \rightarrow 0$, we can study the $\tau$-dependence of the results and perform extrapolation $\tau \rightarrow 0$. In Fig. 3 the $\tau$-dependence of the continuum extrapolated correlator for the temperature $T / T_{c}=1.24$ is presented. One clearly sees that this dependence can be described as linear, what allowed to perform an extrapolation $\tau \rightarrow 0$. Finally, we present the extrapolated correlator of the topological charge density in Fig. 4. 

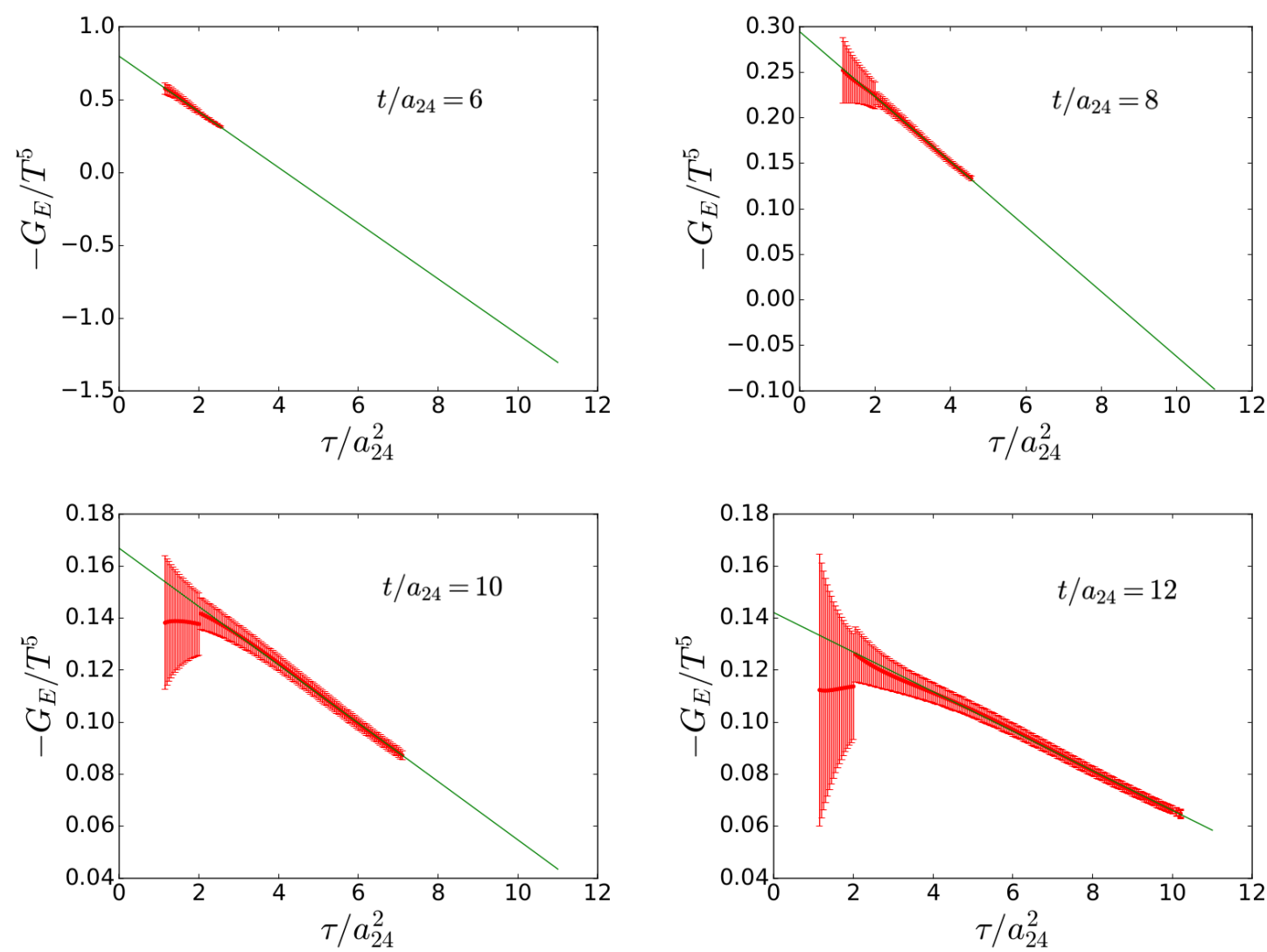

Figure 3: The dependence of the continuum extrapolated correlator on the GF time $\tau$ for four values of the Euclidean time $t / a_{24}=6,8,10,12$.

Now let us discuss the inversion of Eq. 2.2. Since gluodynamics is asymptotically free, at large frequencies the behaviour of $\rho(\omega)$ should be described by the perturbation theory. Leading order results for $\rho(\omega)$ :

$$
\rho^{L O}(\omega)=-\frac{d_{A} \alpha_{s}^{2}}{256 \pi^{4}} \omega^{4}
$$

where $d_{A}=8$ is the number of gluons. Note that behaviour is $\sim \omega^{4}$, but numerical coefficient is very small. To estimate the contribution of this UV tail, we convoluted the spectral function $\rho^{L O}(\omega) \theta\left(\omega-\omega_{0}\right)$ with the kernel (2.2). The threshold $\theta_{0}$ was taken to be 5T. This contribution is also shown in Fig. 4. One clearly sees that for all points under considerations the contribution of the ultraviolet part is at least by order of magnitude smaller, which makes the problem of inversion of (2.2) for sphaleron rate not so difficult as, i.e., for the shear viscosity [12].

To invert the integral relation (2.2) we used Backus-Gilbert method[19, 20]. In this method one obtains an estimator $\hat{\rho}$ of the spectral function $\rho$ according to: $\hat{\rho}(\omega)=\sum_{i} G_{i} u_{i}(\omega)$, where $G_{i}=G_{E}\left(t_{i}\right)$ is the data for the correlator and $u_{i}(\omega)$ are some functions. If one takes the expression (2.2) for the points $G_{i}=G_{E}\left(t_{i}\right)$, then $\rho$ and $\hat{\rho}$ are related via $\hat{\rho}(\bar{\omega})=\int_{0}^{\infty} d \omega \hat{\delta}(\bar{\omega}, \omega) \rho(\omega)$. 


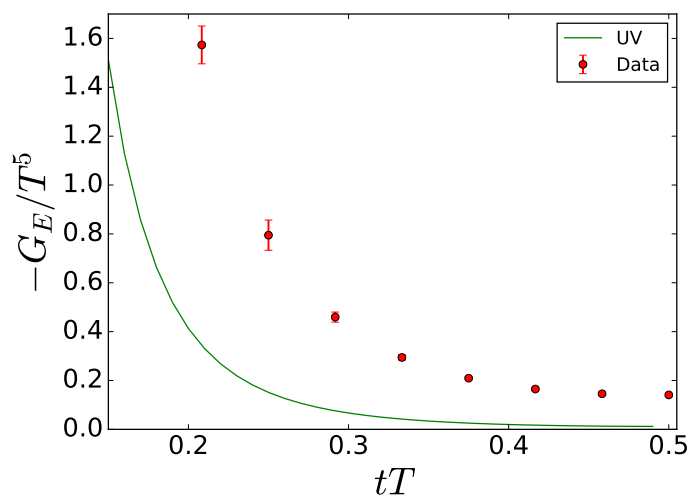

Figure 4: Renormalized correlator of the topological charge density as a function of Euclidean time for temperature $T / T_{c}=1.24$ (red points). Green line corresponds to the contribution of the ultraviolet part of the spectral function.

$\hat{\delta}(\bar{\omega}, \omega)=\sum_{i} K\left(\omega, t_{i}\right) u_{i}(\bar{\omega})$ is called a resolution function. The functions $u_{i}(\omega)$ are chosen in such way that $\hat{\delta}(\bar{\omega}, \omega)$ is close to delta function $\delta(\bar{\omega}-\omega)$, then $\hat{\rho}(\omega)$ is a good estimation of $\rho(\omega)$. The exact way of choosing these function is described, i.e., in [12]. It is important to note that this method contains additional parameter - a regulator $\lambda$. By varying $\lambda$ one can decrease the final statistical error at the price of making the resolution function $\delta$ wider. By changing this parameter we estimated systematical uncertainty of the final result. Statistical error is much smaller then systematical.

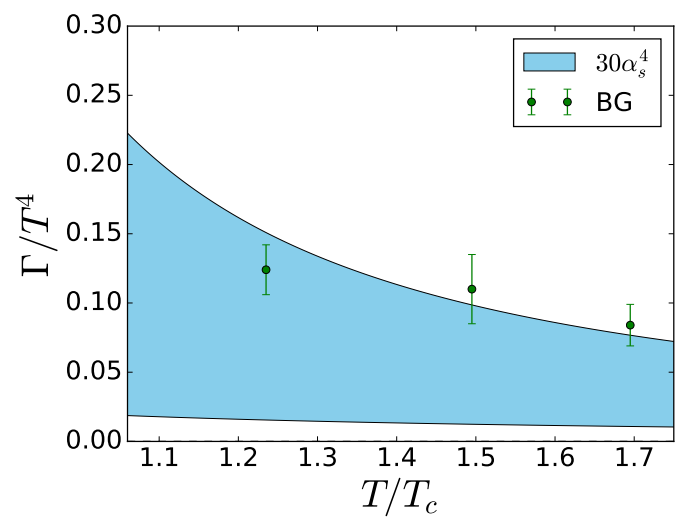

Figure 5: The dependence of the sphaleron rate on the temperature $T / T_{c}$. Green points correspond to our results. Blue band represents the predictions of [5].

The resulting dependence of the reconstructed sphaleron rate $\Gamma$ on the temperature of the system is shown on Fig. 5. On the same plot we showed the predictions of [5]. To estimate the coupling constant $\alpha_{s}$ as a function of temperature we used two-level formula with the scale given by $\mu \sim(1-4) \pi T$. It can be seen that our results are close to those of [5], although seem to favour 
larger values.

In conclusion, we measured the correlator of the topological charge density in gluodynamics in the deconfinement phase using Gradient Flow. The results are extrapolated to the continuum. Using the extrapolated correlator we estimated the sphaleron rate in gluodynamics and its temperature dependence. The results are in agreement with the estimation of [5].

\section{Acknowledgments}

The authors are grateful to V.V. Braguta, N.Yu. Astrahantsev and E.-M. Ilgenfritz for interesting and useful discussion. This work has been carried out using computing resources of the federal collective usage center Complex for Simulation and Data Processing for Mega-science Facilities at NRC "Kurchatov Institute", http://ckp.nrcki.ru/. Part of the simulations has been performed on the supercomputer of NRC "Kurchatov Institute" - ITEP. The work was supported by RFBR grant 18-02-01107.

\section{References}

[1] Curtis G. Callan, Jr., R. F. Dashen, and David J. Gross. The Structure of the Gauge Theory Vacuum. Phys. Lett., B63:334-340, 1976. [,357(1976)].

[2] R. Jackiw and C. Rebbi. Vacuum Periodicity in a Yang-Mills Quantum Theory. Phys. Rev. Lett., 37:172-175, 1976. [,353(1976)].

[3] A. A. Belavin, Alexander M. Polyakov, A. S. Schwartz, and Yu. S. Tyupkin. Pseudoparticle Solutions of the Yang-Mills Equations. Phys. Lett., B59:85-87, 1975. [,350(1975)].

[4] Dietrich Bodeker. On the effective dynamics of soft nonAbelian gauge fields at finite temperature. Phys. Lett., B426:351-360, 1998.

[5] Guy D. Moore and Marcus Tassler. The Sphaleron Rate in SU(N) Gauge Theory. JHEP, 02:105, 2011.

[6] Gokce Basar and Dmitri E. Kharzeev. The Chern-Simons diffusion rate in strongly coupled N=4 SYM plasma in an external magnetic field. Phys. Rev., D85:086012, 2012.

[7] U. Gürsoy, I. Iatrakis, E. Kiritsis, F. Nitti, and A. O'Bannon. The Chern-Simons Diffusion Rate in Improved Holographic QCD. JHEP, 02:119, 2013.

[8] Ernst-Michael Ilgenfritz and H. Panagopoulos. Towards a topological transition rate from high temperature lattice gauge theory. Phys. Lett., B258:415-420, 1991.

[9] Gert Aarts, Chris Allton, Alessandro Amato, Pietro Giudice, Simon Hands, and Jon-Ivar Skullerud. Electrical conductivity and charge diffusion in thermal QCD from the lattice. JHEP, 02:186, 2015.

[10] D. L. Boyda, V. V. Braguta, M. I. Katsnelson, and M. V. Ulybyshev. Many-body effects on graphene conductivity: Quantum Monte Carlo calculations. Phys. Rev., B94(8):085421, 2016.

[11] Harvey B. Meyer. A Calculation of the shear viscosity in SU(3) gluodynamics. Phys. Rev, D76:101701, 2007.

[12] N. Astrakhantsev, Viktor Braguta, and Andrey Kotov. Temperature dependence of shear viscosity of SU(3)-gluodynamics within lattice simulation. JHEP, 04:101, 2017. 
[13] N. Yu. Astrakhantsev, V. V. Braguta, and A. Yu. Kotov. Study of shear viscosity of SU(2)-gluodynamics within lattice simulation. JHEP, 09:082, 2015.

[14] Harvey B. Meyer. A Calculation of the bulk viscosity in SU(3) gluodynamics. Phys. Rev. Lett., 100:162001, 2008.

[15] N. Yu. Astrakhantsev, V. V. Braguta, and A. Yu. Kotov. Temperature dependence of bulk viscosity within lattice simulation of $S U(3)$-gluodynamics. 2018.

[16] Martin Lüscher. Properties and uses of the Wilson flow in lattice QCD. JHEP, 08:071, 2010. [Erratum: JHEP03,092(2014)].

[17] Marco Cè, Cristian Consonni, Georg P. Engel, and Leonardo Giusti. Non-Gaussianities in the topological charge distribution of the SU(3) Yang-Mills theory. Phys. Rev., D92(7):074502, 2015.

[18] Masakiyo Kitazawa, Takumi Iritani, Masayuki Asakawa, Tetsuo Hatsuda, and Hiroshi Suzuki. Equation of State for SU(3) Gauge Theory via the Energy-Momentum Tensor under Gradient Flow. Phys. Rev., D94(11):114512, 2016.

[19] George Backus and Freeman Gilbert. The resolving power of gross earth data. Geophysical Journal International, 16(2):169-205, 1968.

[20] G. Backus and F. Gilbert. Uniqueness in the inversion of inaccurate gross earth data. Philosophical Transactions of the Royal Society of London A: Mathematical, Physical and Engineering Sciences, 266(1173):123-192, 1970. 